\title{
Understanding and Designing Embodied Experiences Through Mid-air Tactile Stimulation
}

\author{
Dario Pittera \\ Sussex House, BN19RH \\ Brighton, UK \\ d.pittera@sussex.ac.uk
}

\begin{abstract}
Current attempts to render touch in multimedia technology still represents a challenge. Touch is indeed a complex system and there are many aspects to take into account when trying to rendering it (e.g. the compliance of an object, its weight, orientation, geometric properties and forces on the skin). This is especially true for VR, where touch is an important factor to achieve the embodiment in virtual environments. Recently, new tactile technology has been developed: the mid-air devices, capable of delivering tactile feedback without entering in contact with the skin. One of the contributions of the doctoral research described in this paper is to overcome design challenges and create immersive experiences by applying psychological principles and paradigms, exploiting the advantages of the mid-air technology. We designed possible embodied interaction scenarios in mid-air and physical touch. Findings from these research point to opportunities for designing new immersive experiences. Future work will involve different parts of the body and different tactile properties (e.g. thermal stimulation).
\end{abstract}

\section{Author Keywords}

Touch; Mid-air; Embodiment; Virtual reality; VR; Haptics; User Experiences; Multisensory design.

\section{CONTENT AND MOTIVATION}

Touch is a complex sense that conveys information about pressure, pain, proprioception, movement, temperature, and pleasure (through the C-tactile fibers) [9, 1]. Designing for tactile experiences has become of primary interest in the field of HCI and the design of new technologies; e.g. the Disney AIREAL exploring mid-air touch through vortexes by Sodhi et al. [17], the laser approach to create tactile feedback by

Lee et al. [12], the Ultrahaptics device, employing ultrasounds by Carter et al. [7] or the fingertip tactile device by Schorr and Okamura [15]. Interactive technologies are increasingly exploiting touch and implementing tactile feedback to provide

Permission to make digital or hard copies of all or part of this work for personal or classroom use is granted without fee provided that copies are not made or distributed for profit or commercial advantage and that copies bear this notice and the full citation on the first page. Copyrights for components of this work owned by others than ACM must be honored. Abstracting with credit is permitted. To copy otherwise, or republish, to post on servers or to redistribute to lists, requires prior specific permission and/or a fee. Request permissions from permissions@acm.org.

TEI 2018, March 18-21, 2018, Stockholm, Sweden.

Copyright () 2018 ACM ISBN 978-1-4503-5568-1/18/03 ...\$15.00.

http://dx.doi.org/10.1145/3173225.3173340

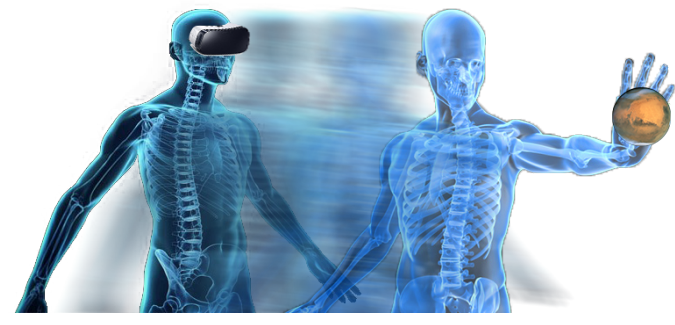

Figure 1. Designing embodied experiences in VR.

the user with an additional information channel in the interaction with devices, products, and services (e.g. Oculus and HTC Touch Controllers, Apple Touch). Most recently, mid-air haptics technology (i.e., devices that enable the creation of contactless tactile feedback in mid-air) represents an exciting new approach to convey tactile feedback to users.

While various mid-air devices are becoming available on the consumer market, the ones that employ focalized ultrasounds to deliver a tactile feedback on the users' bare hands, are the most promising. The ultrasound technology has a greater temporal and spatial resolution (1 $\mathrm{cm} \mathrm{[13])} \mathrm{compared} \mathrm{to} \mathrm{vortex}$ based and compressed air systems, it allows to design complex patterns (static and dynamic) [13], and it is also safer than, for instance, laser-based approaches. Advantages of mid-air technology compared to other touch interfaces is that they allow users to interact without any physical attachment to the body and they can provide fast changing tactile patterns. In the realm of HCI and interaction design, we can exploit these advantages to create new scenarios, especially relevant in the design of VR experiences. The current challenge in $\mathrm{VR}$ is to make experiences and interactions more realistic and immersive. However, current efforts to go beyond audio-visual stimulation, involving the sense of touch, are limited due to the challenge of rendering tactile sensations in a compelling way.

The ambition of this Ph.D. project is to contribute to the challenge of creating convincing and immersive embodied experiences through tactile stimulation. We are particularly interested in the systematic investigation of mid-air tactile stimulation to created embodied experiences. Defining the embodiment is not straightforward as one would hope [8, 14]. Carruthers [6] defines the sense of embodiment as an offline representation of the body; what we usually think of as our body, opposed to what our body looks like moment by moment. 
The sense of embodiment mainly consists of two components: 1) the feeling of being distinct from other objects and people [5]; 2) the fact that everyone experiences the world in the first-person perspective. A human being is a fusion between the mind and the body, and this is located in an environment, that is also social and with whom it acts and interacts [4]. The embodiment is telling us what is to be considered self-related and what it is the external world. In essence, the sense of embodiment can be defined as the system that makes us distinguish ourselves from the rest (the world, the other people); it is the perception of our physical boundaries.

\section{RESEARCH OBJECTIVES AND QUESTIONS}

Mid-air devices are relatively new and an unexploited design space. There are still various unanswered questions and challenges on how mid-air stimulation can be used to create new experiences. This Ph.D. aims to contribute to this emerging field and understanding of mid-air technology in HCI through increasing the understanding of such technology for experience design especially in the context of a users' sense of embodiment when interacting within a virtual environment. The main objectives are:

1. Explore the users' body sensitivity to ultrasound stimulation. Where can people perceive mid-air stimulation?

2. Apply psychological paradigms to test the occurrence of bodily illusions in real and virtual environments. How well does mid-air touch work in relation to physical touch creating embodied experiences?

3. Design specific application scenarios integrating tactile stimulation with other sensory stimuli (vision and sound) to create interactive embodied experiences, especially in VR. How will the brain integrate different sensory stimuli?

\section{METHOD AND PRELIMINARY RESULTS}

In order to address the above research challenges and objectives, we draw upon well-established principles and paradigms in psychology such as the rubber hand illusion [3], the body transfer illusion [16], or the out of body experiences [2]. These paradigms are being studied by means of physical touch and it is not clear if mid-air technology is capable of delivering the same percept. Therefore, we think that exploiting specific psychological paradigms in combination with mid-air touch in comparison to physical touch could provide novel insights and advancement for designing immersive and embodied interaction experiences in HCI.

More specifically, to address objective 1 we use principles of psychophysics methodology. The psychophysics studies the minimum quantity of stimulus needed for a human to recognize, feel, the stimulus. The main techniques are the method of constant stimuli, the staircase procedure and the method of limits [11]. The most indicative measurements in psychophysics are usually the detection thresholds and the just noticeable difference JND [10]. The first measure provides researchers with the minimum stimulus intensity a human (or animal) can detect, the second one, is the smallest change in a stimulus which a person can detect $50 \%$ of the time. These thresholds are known to vary depending on the area of stimulation [18]. Therefore, knowing these psychophysical parameters allows us to design optimal systems in tune with our perception ability. Preliminary results: not the entire surface of the body skin is sensitive to mid-air stimulation. We believe that the main explanation dwells in the lacking of Pacinian corpuscles. We explore the body starting from the arm and the hand. The main results are illustrated in Figure 2.

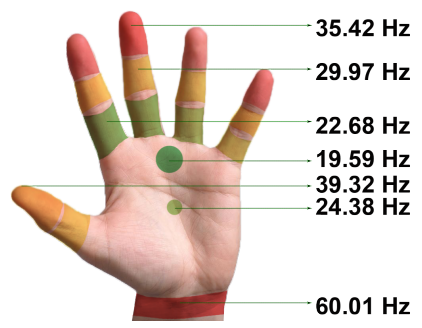

Figure 2. Psychophysics results for mid-air stimulation.

Moreover, to address objective 2 we employed a typical paradigm used for the study of embodiment and to demonstrate the plasticity of our body schema: the rubber hand illusion (RHI) [3]. The subject sits with the real hand covered and out of the sight, while a rubber hand is located in front of him/her. By stroking the fake hand synchronized with the real one, it is possible to induce the illusion that the fake hand is actually the real one. Preliminary results: we replicated the RHI paradigm in VR employing mid-air touch. Classically the experiment consists of two conditions: one synchronous stimulation and one asynchronous stimulation where the illusion is broken. We added another condition: incongruent but with multiple stimuli to investigate if the same illusion could be achieved. We illustrated that it is possible to create the illusion mediated by mid-air touch and that the illusion can be reached even in an incongruent condition.

Finally, to address objective 3 , we investigated the visualtactile integration in a virtual environment. In the realm of psychology's constructs, tactile illusions appear to be another tool we can potentially use in our research. Hence, we exploited the apparent tactile movement illusion. Two actuators are activated and the stimulus-onset asynchrony (SOA) is modulated so that the user will perceive a feeling of movement between the two sites of stimulation. Only when the SOA is optimal the two vibrations appear as a continuous movement. Preliminary results: We explored the tactile illusion of movement between the two hands. We calculated the optimal onset time between the two actuators. We then observed the different time perception when tactile and visual cues are integrated.

\section{PLANNED ACTIVITIES}

I will deepen the illusion of movement in mid-air and I will implement thermal stimuli in my future studies.

We believe that the outcomes of our research could be useful for scientists and designers who want to create new embodied experiences to an always more demanding public.

\section{REFERENCES}

1. Wouter M Bergmann Tiest and Astrid M.L. Kappers. 2006. Analysis of haptic perception of materials by 
multidimensional scaling and physical measurements of roughness and compressibility. Acta Psychologica 121, 1 (2006), 1-20. DOI :

http://dx.doi.org/10.1016/j.actpsy.2005.04.005

2. Olaf Blanke, Theodor Landis, Laurent Spinelli, and Margitta Seeck. 2004. OutâĂ ̌̌ofâĂ̌̌body experience and autoscopy of neurological origin. Brain 127, 2 (2 2004), 243-258. DOI : http://dx.doi.org/10.1093/brain/awh040

3. M. Botvinick and J. Cohen. 1998. Rubber hands 'feel' touch that eyes see. Nature 391, 6669 (1998), 756. DOI : http://dx.doi.org/10.1038/35784

4. Peter Brugger and Bigna Lenggenhager. 2014. The bodily self and its disorders. Current Opinion in Neurology 27, 6 (2014), 644-652. DOI : http://dx.doi.org/10.1097/WC0.0000000000000151

5. Glenn Carruthers. 2007. A model of the synchronic self. Consciousness and Cognition 16, 2 (2007), 533-550. DOI : http://dx.doi .org/10.1016/j. concog.2006.06.002

6. Glenn Carruthers. 2008. Types of body representation and the sense of embodiment. Consciousness and Cognition 17, 4 (2008), 1302-1316. DOI: http://dx.doi.org/10.1016/j.concog.2008.02.001

7. Tom Carter, Sue Ann Seah, Benjamin Long, Bruce Drinkwater, and Sriram Subramanian. 2013. UltraHaptics. Proceedings of the 26th annual ACM symposium on User interface software and technology - UIST'13 (2013), 505-514. DOI : http://dx.doi.org/10.1145/2501988.2502018

8. Arthur M. Glenberg. 2010. Embodiment as a unifying perspective for psychology. Wiley Interdisciplinary Reviews: Cognitive Science (2010), n/a-n/a. DOI : http://dx.doi.org/10.1002/wcs. 55

9. David Katz and Lester E. Krueger. World of touch. 260 pages.
10. Frederick A. A. Kingdom and Nicolaas Prins. 2010. Psychophysics : a practical introduction. Elsevier/Academic Press. 279 pages.

11. Frederick a a Prins Kingdom. 2010. Psychophysics: $a$ pratical introduction. 297 pages.

12. Hojin Lee, Ji-sun Kim, Seungmoon Choi, Jae-hoon Jun, A-hee Kim, Han-byeol Oh, Jong-rak Park, Hyung-sik Kim, and Soon-cheol Chung. 2015. Mid-Air Tactile Rendering Using Laser-Induced Thermoelastic Effects : The First Study for Indirect Radiation. (2015).

13. Benjamin Long, Sue Ann Seah, Tom Carter, and Sriram Subramanian. 2014. Rendering volumetric haptic shapes in mid-air using ultrasound. ACM Transactions on Graphics 33, 6 (2014), 1-10. DOI: http://dx.doi.org/10.1145/2661229.2661257

14. Matthew R. Longo, Friederike Schüür, Marjolein P.M. Kammers, Manos Tsakiris, and Patrick Haggard. 2008. What is embodiment? A psychometric approach. Cognition 107, 3 (2008), 978-998. D0I : http://dx.doi.org/10.1016/j.cognition.2007.12.004

15. Samuel B Schorr and Allison M Okamura. 2017. Fingertip Tactile Devices for Virtual Object Manipulation and Exploration. In Proceedings of the 2017 CHI Conference on Human Factors in Computing Systems CHI'17. 3115-3119. DOI : http://dx.doi.org/10.1145/3025453.3025744

16. Mel Slater, Bernhard Spanlang, Maria V. Sanchez-Vives, and Olaf Blanke. 2010. First person experience of body transfer in virtual reality. PLOS ONE 5, 5 (2010), 1-9. DOI : http://dx.doi .org/10.1371/journal . pone.0010564

17. R Sodhi, Poupyrev, M Glisson, and a Israr. 2013. AIREAL: interactive tactile experiences in free air. $A C M$ Transactions on Graphics 32, 4 (2013), 134. DOI : http://dx.doi.org/10.1145/2461912.2462007

18. S. Weinstein. 1968. Intensive and Extensive Aspects of Tactile Sensitivity as a Function of Body Part, Sex and Laterality. In Proc. 1st Int. Symp. on the Skin Senses. 